Check for updates

Cite this: Chem. Sci., 2020, 11, 343

๑ All publication charges for this article have been paid for by the Royal Society of Chemistry
Received 1st October 2019

Accepted 5th November 2019

DOI: $10.1039 / c 9 s c 04950 a$

rsc.li/chemical-science

\section{Manipulating molecules with strong coupling: harvesting triplet excitons in organic exciton microcavitiest:}

\author{
Daniel Polak, ${ }^{a}$ Rahul Jayaprakash, ${ }^{a}$ Thomas P. Lyons, (D) a Luis Á. Martínez- \\ Martínez, (D) ${ }^{\mathrm{b}}$ Anastasia Leventis, ${ }^{\mathrm{c}}$ Kealan J. Fallon, ${ }^{\mathrm{C}}$ Harriet Coulthard, ${ }^{a}$ \\ David G. Bossanyi, (D) ${ }^{a}$ Kyriacos Georgiou, ${ }^{a}$ Anthony J. Petty, II, ${ }^{a}$ John Anthony, (ID)d \\ Hugo Bronstein, (D) ${ }^{c}$ Joel Yuen-Zhou, (D) ${ }^{b}$ Alexander I. Tartakovskii, ${ }^{a}$ Jenny Clark (DD *a \\ and Andrew J. Musser $\mathbb{D}$ *ae
}

\begin{abstract}
Exciton-polaritons are quasiparticles with mixed photon and exciton character that demonstrate rich quantum phenomena, novel optoelectronic devices and the potential to modify chemical properties of materials. Organic materials are of current interest as active materials for their ability to sustain excitonpolaritons even at room temperature. However, within organic optoelectronic devices, it is often the 'dark' spin-1 triplet excitons that dominate operation. These triplets have been largely ignored in treatments of polaritons, which instead only consider the role of states that directly and strongly interact with light. Here we demonstrate that these 'dark' states can also play a major role in polariton dynamics, observing polariton population transferred directly from the triplet manifold via triplet-triplet annihilation. The process leads to polariton emission that is longer-lived ( $>\mu \mathrm{s})$ even than exciton emission in bare films. This enhancement is directly linked to spin-2 triplet-pair states, which are formed in films and microcavities by singlet fission or triplet-triplet annihilation. Such high-spin multiexciton states are generally non-emissive and cannot directly couple to light, yet the formation of polaritons creates for them entirely new radiative decay pathways. This is possible due to weak mixing between singlet and triplet-pair manifolds, which - in the strong coupling regime - enables direct interaction between the bright polariton states and those that are formally non-emissive. Our observations offer the enticing possibility of using polaritons to harvest or manipulate population from states that are formally dark.
\end{abstract}

\section{Introduction}

The exploration of new material properties typically faces significant practical constraints from cumbersome synthesis and morphological control. In recent years, however, it has been shown that many materials properties can be non-synthetically

${ }^{a}$ Department of Physics and Astronomy, University of Sheffield, Hicks Building, Hounsfield Road, Sheffield S3 7RH, UK. E-mail: jenny.clark@sheffield.ac.uk

${ }^{b}$ Department of Chemistry and Biochemistry, University of California San Diego, La Jolla, California 92093, USA

'Department of Chemistry, University of Cambridge, Lensfield Road, Cambridge CB2 $1 E W, U K$

${ }^{d}$ Department of Chemistry, University of Kentucky, Lexington, Kentucky 40506-0055, USA

${ }^{e}$ Department of Chemistry and Biochemistry, Cornell University, Ithaca, New York 14853,USA.E-mail:ajm557@cornell.edu

$\dagger$ The data underlying all figures in this publication are available at https://figshare.com/s/59d2dc8215881c9a157d

\$Electronic supplementary information (ESI) available: Materials and experimental methods, reference sample characterisation, detailed kinetic model. See DOI: 10.1039/c9sc04950a tuned with confined light fields to form exciton-polaritons ${ }^{1-3}$ or vibro-polaritons, ${ }^{4-6}$ pointing the way to an entirely new field of microcavity-controlled chemistry. ${ }^{7-11}$ These exciton-polaritons are quasi-particles mixing light (photon) and matter (exciton) components, leading to rich quantum effects ${ }^{12-16}$ and potential optoelectronic applications., ${ }^{2,3,7,17-21}$ Exciton-polaritons are formed by placing a semiconductor or dye between two mirrors to create a Fabry-Perot microcavity in which light of the correct angle and wavelength can be trapped (Fig. 1a). If the material within the cavity has a strong exciton absorption, in resonance with the trapped photon mode, the exciton and photon can couple and form hybrid polariton states (Fig. 1b). As a consequence of the mixed exciton-photonic character of these states, a measurement of reflected light as a function of incident angle demonstrates the typical dispersion shown in Fig. 1c, with the upper polariton branch (UPB) and lower polariton branch (LPB) split around the excitonic energy.

Most studies of exciton-polariton physics have focussed on inorganic semiconductor systems..$^{12,13,16-18}$ In comparison, organic materials have the advantage of high oscillator strength, ${ }^{22}$ affording much stronger light-matter coupling that 
a

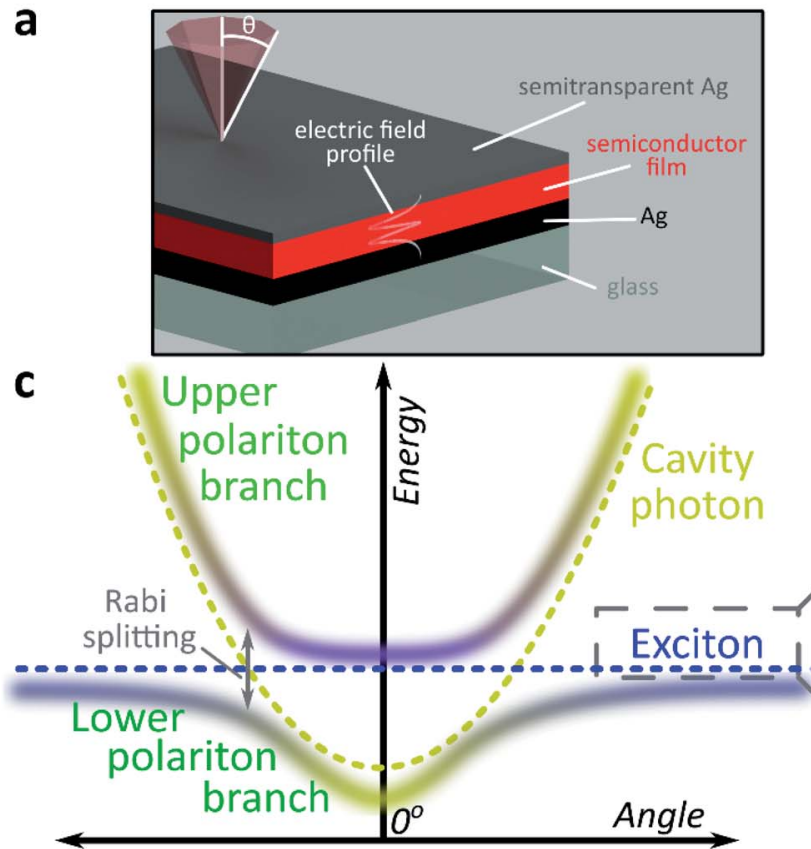

b Upper polariton

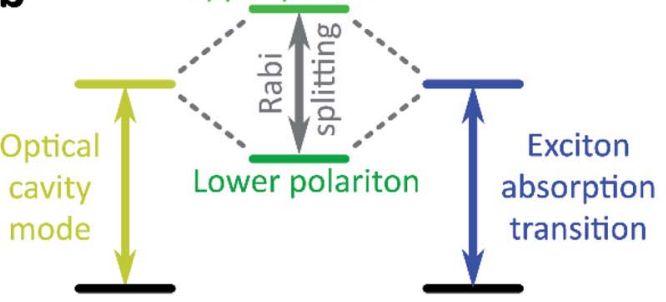

d

Intracavity exciton dynamics

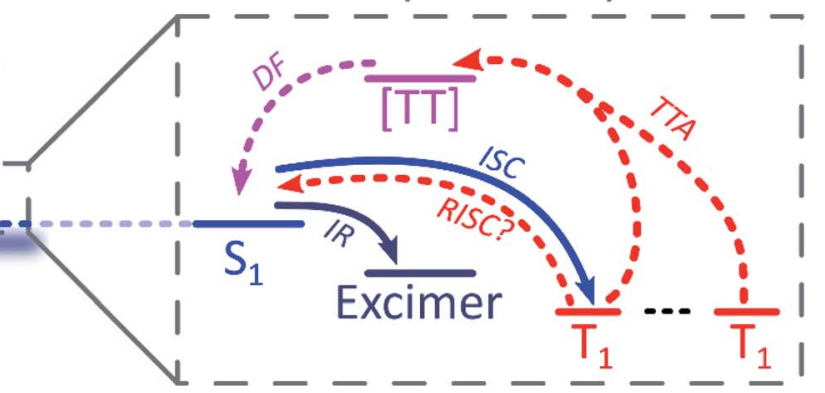

Fig. 1 Strong light-matter coupling in optical microcavities. (a) Microcavity structure. A thin film of organic semiconductor or dye dispersed in neutral polymer matrix is deposited in the cavity defined by the two mirrors, here Ag. The thickness of the cavity determines the energy of the confined photonic mode and thus the profile of the electric field inside the cavity, shown here for the $\lambda$-mode. Reflection and emission from the cavity are measured as a function of angle $\theta$, with $0^{\circ}$ defined as normal to the cavity surface. (b) When the cavity mode and the excitonic transition of the semiconductor are near resonance, these two states can couple, forming hybrid upper and lower polariton states. (c) Unlike the exciton (blue), the cavity mode (gold) exhibits distinct angular dispersion. Coupling between the two yields dispersed polariton branches, with characteristic anti-crossing at the exciton energy. Shading indicates the degree of photonic (gold) vs. excitonic (blue) character in the state. (d) Typical excitonic processes possible within organic semiconductor films. IR: intermolecular relaxation, (R)ISC: (reverse) intersystem crossing, TTA: triplet-triplet annihilation, DF: delayed fluorescence. Solid arrows indicate processes known to modify exciton-polariton emission dynamics, while dashed arrows show processes not typically explored within microcavities.

can result in Rabi splittings between the UPB and LPB in the range $0.1-1 \mathrm{e} \quad \mathrm{V}^{\mathbf{1 , 2 , 2 2 - 2 5}}$ Organics also have low dielectric constants $\left(\varepsilon_{\mathrm{r}}\right.$ typically $\left.2-4\right)$. Consequently, photoexcitation results in bound electron-hole pairs known as Frenkel excitons, with binding energies on the order of $0.5-1 \mathrm{eV}$. Such high binding energies mean exciton-polaritons are stable and can even condense at room temperature; the latter phenomenon has been observed now in several organic semiconductor

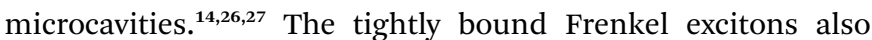
exhibit complex photophysics, with numerous radiative and non-radiative decay pathways possible following initial photoexcitation (Fig. 1d). ${ }^{28}$ These pathways are also active within microcavities, specifically in the 'exciton reservoir' of uncoupled intracavity states, and it is typically expected that they proceed similarly to in free films. They are rarely treated in detail in organic exciton-polariton studies, where the focus is primarily on 'bright' singlet (spin-0) excitons. However, it has been shown that the polariton emission dispersion and dynamics can be significantly influenced by population transfer from the reservoir - the parent bright state ${ }^{29-31}$ or weakly emissive excimers formed by intermolecular relaxation ${ }^{32,33}$ - and theoretical attention increasingly has started to turn to the impact of other non-radiative photophysical processes. ${ }^{9-11,34}$

We focus here on the role in these systems of triplet (spin-1) excitons. An additional consequence of the low dielectric constant of organic materials is a large exchange energy, which results in the lowest triplet exciton being located $>0.5 \mathrm{eV}$ below the first singlet exciton. ${ }^{35}$ Triplet excitons and their management are critical in organic semiconductor devices such as displays and solar cells. ${ }^{35-38}$ For example, $75 \%$ of excitons formed by electron-hole recombination in optoelectronic devices are triplets due to spin statistics. Triplets are a main reason for the absence of continuous optically pumped organic lasers, but could be useful in solar cells..$^{37,38}$ Triplets can be generated from singlet excitons via intersystem crossing, which is generally slow (ns or longer) due to weak spin-orbit coupling. Once formed, return to the ground-state requires a spin-flip. Therefore, triplets cannot be directly photoexcited and, in typical organic materials, are non-emissive and long-lived $(\gg \mu s)$.

Only states with large oscillator strength couple to the photon in a microcavity, with triplet states considered a loss channel in organic exciton-polariton systems. ${ }^{25}$ However, states within the reservoir - including triplets, as we demonstrate here - can interact with the polaritons. It has recently been shown that polaritons can be used to alter the decay lifetime of weakly emissive triplet states, and it was suggested this was due to efficient reverse intersystem crossing from the triplets into the lower polariton. ${ }^{39}$ A caveat in that study is that transfer into the lower polariton was not directly resolved, and subsequent 
theoretical and experimental work indicates that, for a broad range of materials, this process could be less efficient than the intrinsic reverse intersystem crossing channel..$^{\mathbf{4 0 4 1}}$ Moreover, both experimental studies have focused on materials where the triplet state is already partially emissive. ${ }^{39,40}$ Similarly, a recent report on tetracene single crystals studied delayed emission caused by triplet-triplet annihilation. ${ }^{\mathbf{4 2}}$ This process is well known in the bulk material and results in substantial emission from the reservoir of triplet states. ${ }^{\mathbf{4 3 , 4 4}}$ Upon strong coupling to localised surface plasmon resonances the crystal exhibited an enhancement of both prompt and delayed emission in the strong-coupling regime. ${ }^{42}$ The mechanism of this effect is unclear, though the facile conversion between singlet and triplet manifolds suggests that radiative pumping ${ }^{32}$ may play a role. Significantly more work is needed to understand if and how 'dark' triplet states - the most common type in organic semiconductors - can interact with the polariton. This problem takes on particular importance in light of the continued progress in electrically injected polariton devices, ${ }^{\mathbf{1 8 - 2 0}}$ where triplets can no longer be ignored. Just like with electrical injection, a very large reservoir of triplets can be generated by photoexcitation in some materials, making them excellent model systems for the detailed study of polariton-triplet interactions. Large triplet populations can be optically generated in materials with strong spin-orbit coupling resulting in fast intersystem crossing $^{25}$ or in materials in which the exchange energy is so large that the singlet energy is approximately twice the triplet energy. In the latter, photoexcitation into the singlet state results in formation of two triplets through singlet exciton fission. ${ }^{28,45}$

We use both intersystem crossing and singlet fission to optically generate triplets and show how triplet excitons interact with polariton states. We find that strong coupling creates entirely new radiative channels that are unavailable in the bare film. The microcavity allows us to extract light from these 'dark' triplet states, 'tilting' the system towards photon emission in an excitonic analogue to the tuning of chemical reactivity enabled by strong-coupling to molecular vibrational transitions. ${ }^{6}$ This results in ultra-long-lived polariton emission and the potential for harvesting 'dark' triplets in devices. We propose a mechanism based on population transfer between the different state manifolds and the new pathways that emerge under strong coupling, and we show that even though the dynamics are dominated by transfer between the dark triplet and singlet states at early times, an efficient channel of conversion of triplet states to light-emitting polariton modes is predominant at long times. The small but non-zero coupling between these dark excitonic states and the polariton stems from the widespread phenomenon of excited-state mixing, which could open the way to using strong light-matter coupling to manipulate the dynamics of states that do not interact directly with light.

\section{Results}

\section{Polariton emission from triplet-triplet annihilation}

A common way to study triplet excitons is through delayed fluorescence, which can occur through the spin-allowed conversion of two triplets into one singlet exciton, known as 'triplet-triplet annihilation' ${ }^{28,46}$ The resulting emission approximately tracks the $\sim \mu$ s lifetime of the triplet population rather than the ('prompt') $\sim$ ns singlet lifetime. One of the bestcharacterised triplet-triplet annihilation systems is the blend of diphenylanthracene and metal porphyrins used for photon upconversion, ${ }^{47,48}$ as shown in Fig. 2. We depict the photophysics of this system schematically in Fig. 2b: directly exciting the Ptporphyrin at $532 \mathrm{~nm}$ initiates efficient intersystem crossing ( $<100 \mathrm{fs}),{ }^{47}$ producing triplets that can transfer to diphenylanthracene where triplet-triplet annihilation produces 'up-converted' delayed fluorescence at a significantly shorter wavelength than the original excitation. In solution, the long triplet lifetimes allow this process to occur with high quantum efficiency. ${ }^{46,49}$

In order to understand how triplets behave in microcavities, we need to study delayed fluorescence in the solid state, rather than solution. We therefore prepared films of diphenylanthracene/Pt-porphyrin/polystyrene blends with a ratio of $50: 1: 15$. The polystyrene is used to aid mixing between the two active materials and reduce film roughness. Films and microcavities were encapsulated in inert atmosphere to protect against oxygen quenching of triplets ${ }^{50}$ (see ESI, $\$$ Methods). The absorption of a control, non-cavity film is shown in Fig. 2c. Emission behaviour of the films is consistent with literature. ${ }^{47,48}$ As expected, excitation within the Pt-porphyrin band (532 nm) produces up-converted diphenylanthracene fluorescence (400$500 \mathrm{~nm}$ ). However, as with other solid-state up-conversion systems, we also observe strong Pt-porphyrin phosphorescence $(650 \mathrm{~nm})$ due to phase separation. ${ }^{47,48}$

Fig. 2c shows a reflectivity map of a microcavity containing the diphenylanthracene blend, as a function of incident angle and wavelength. The dips in microcavity reflectivity never cross the bare exciton energy (blue dashed). This 'anti-crossing' is a signature of strong light-matter coupling and polariton formation, and the absorbing states are thus split into polariton branches. Transfer-matrix modelling based on measured optical parameters confirms strong coupling in this structure to the diphenylanthracene $S_{1}$ state (Fig. 2c, lines and circles). There is no evidence of strong coupling to the much weaker Ptporphyrin absorption (ESI, Fig S1 ). Microcavity emission originates from the lower polariton branch (Fig. 2c, right), whether we excite the strong-coupled diphenylanthracene directly (355 $\mathrm{nm}$, dashed) or the uncoupled Pt-porphyrin (532 $\mathrm{nm}$, shaded). In the latter case photoexcitation generates a reservoir of uncoupled triplet excitons, therefore the microcavity emission must be due to up-conversion through triplettriplet annihilation, similar to the single-crystal tetracene result ${ }^{42}$ but without direct photoexcitation of the material which undergoes strong coupling (here, the DPA dark reservoir of $\mathrm{S}_{1}$ states).

\section{Long-lived triplet-derived emission in microcavities}

We explore how this triplet harvesting process is affected by strong coupling using time-resolved measurements. Because the processes of interest are intrinsically slow, we apply high- 
a
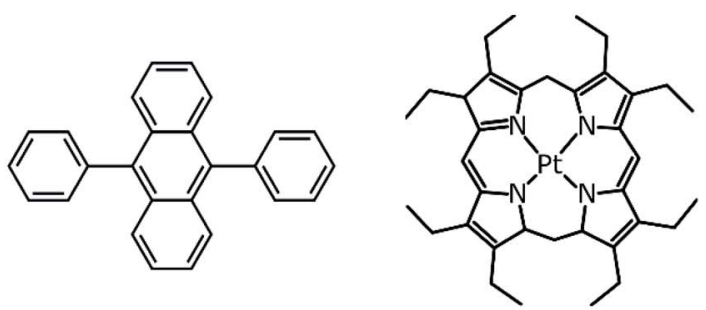

$\begin{array}{cc}\text { 9,10-diphenyl- } & \text { Pt(II) } 2,3,7,8,12,13,17,18- \\ \text { anthracene: } & \text { octaethyl-21H,23H-porphyrin: } \\ \text { DPA } & \text { PtOEP }\end{array}$ b

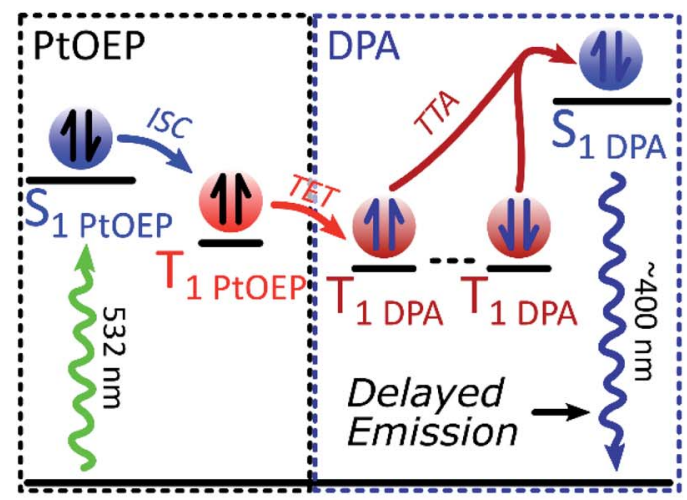

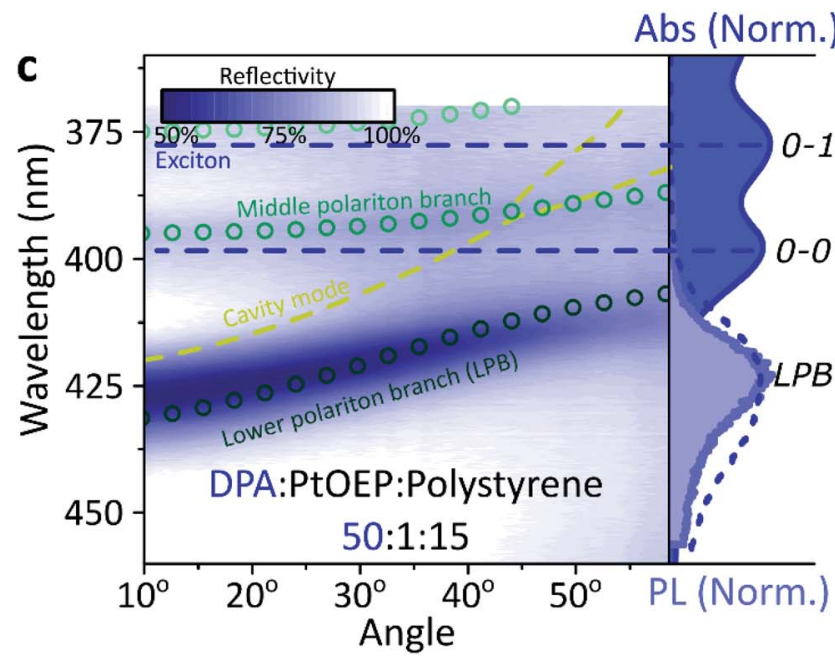

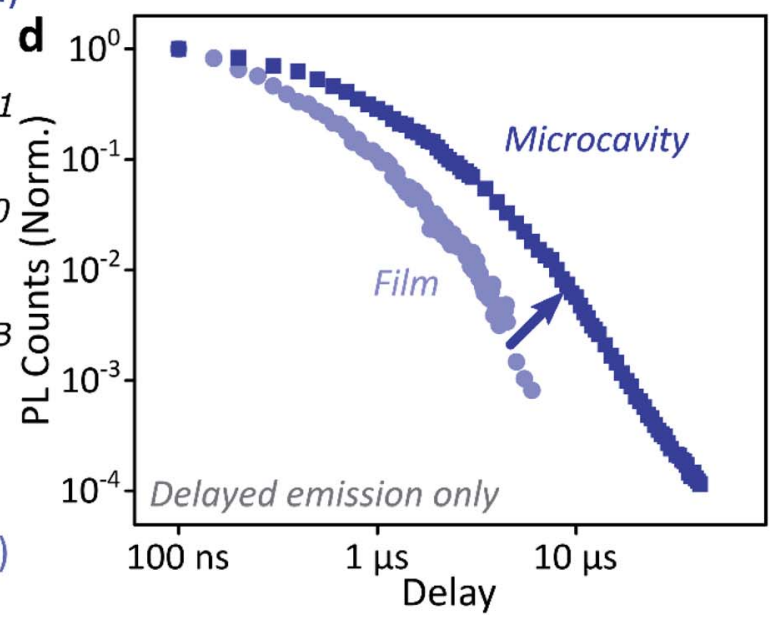

Fig. 2 Sensitised photon up-conversion. (a) Molecular structures of active components used in photon up-conversion system. (b) Simplified schematic of photon up-conversion, details in main text. ISC: intersystem crossing, TET: triplet energy transfer, TTA: triplet-triplet annihilation. (c) Reflectivity map of photon up-conversion blend within a Ag-Ag microcavity. Comparison with absorption spectrum (upper right, dark blue) and transfer matrix modelling (lines, circles) confirms strong coupling, characterised by anti-crossings at the 0-0 and 0-1 energies (dashed). Details of transfer matrix model in ESI, $\$$ Methods. All emission comes from the lower polariton branch (LPB), whether excitation is resonant with diphenylanthracene (355 nm, lower right, dashed spectrum) or PtOEP (532 nm, lower right, shaded spectrum). Emission is collected with a NA = 0.76 lens and thus effectively integrates along the entire dispersion $\left( \pm 45^{\circ}\right)$. (d) Decay kinetics of diphenylanthracene/exciton-polariton emission following excitation of PtOEP at $532 \mathrm{~nm}$ reveal enhanced lifetime in microcavity (dark) vs. bare film (light). All emission on these timescales arises from triplet-triplet annihilation. Incident power (film: $50 \mu \mathrm{W}$, microcavity: $150 \mu \mathrm{W}$ ) yields $\sim 2 x$ greater excitation density in the microcavity, which should result in faster intrinsic depletion of triplets.

sensitivity gated photoluminescence measurements to resolve the dynamics on long timescales. We observe no change in emission lineshape over the full lifetime (ESI, Fig S11 ). After accounting for the differences in absorbed laser power between film and microcavity samples (details in ESI, Section 1.4), we find that the lifetime of emission in the microcavity is distinctly longer than in the film (Fig. 2d). This is the case even though the microcavity is excited at $\sim 2 x$ higher absorbed laser power, resulting in a $2 x$ higher triplet population that, naively, should accordingly undergo faster bimolecular annihilation and exhibit a faster decay of delayed emission. As noted above, all emission on these timescales originates from triplet-triplet annihilation within the reservoir of uncoupled excitons, and we conclude that the microcavity enables harvesting of an additional long-lived species. This change in lifetime is surprising and requires further investigation. We noticed that this ternary blend undergoes laser-induced phase segregation, making detailed studies on this system difficult. We therefore apply the same approach to two simpler reference systems with a single active component.

Diketopyrrolopyrrole thiophene (DPPT, Fig. 3a) is the base unit for polymers exhibiting high charge-carrier mobility in thin-film transistors, recently used in electrical-injection polariton OLEDs. ${ }^{20}$ DPPT monomers are also known to undergo intersystem crossing in the solid state (Fig. $3 \mathrm{~b}$ ). ${ }^{51}$ Films were prepared containing DPPT dispersed in polystyrene matrix (1:4 DPPT : polystyrene). Reference photoluminescence measurements on these films reveal delayed fluorescence which is quenched by oxygen and a non-linear intensity dependence, suggesting the weak delayed fluorescence in DPPT results from bimolecular triplet-triplet annihilation. All subsequent measurements were performed on films or microcavities encapsulated in an oxygen-free environment, unless specifically stated.

Within DPPT-based microcavities, Fig. 3c, we observe a clear anti-crossing at the $0-0$ peak, while the second peak in the 

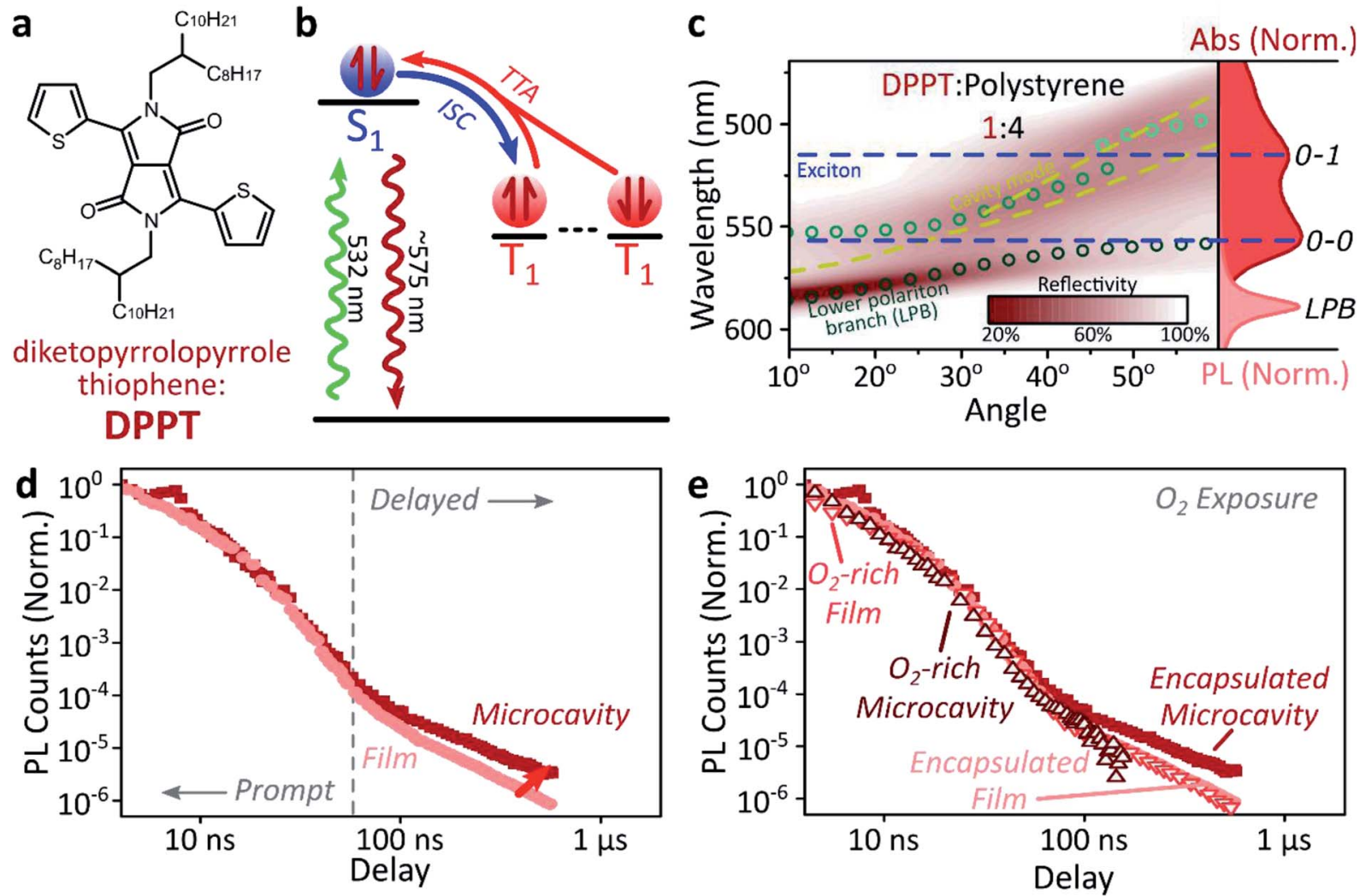

Fig. 3 Delayed emission in single-component film. (a) Molecular structure of DPPT. (b) Simplified schematic of DPPT film photophysics, details in main text. ISC: intersystem crossing, TTA: triplet-triplet annihilation. (c) Reflectivity map of DPPT : polystyrene film within a Ag-Ag microcavity. Comparison with absorption spectrum (right) and transfer matrix modelling (lines, circles) confirms strong coupling to the $0-0$ transition. All emission arises from the lower polariton branch (LPB). Emission is collected with a NA $=0.76$ lens and thus effectively integrates along the entire dispersion $\left( \pm 45^{\circ}\right)$. (d) Integrated photoluminescence kinetics over full emission band for bare film (light) and microcavity (dark) following excitation at $532 \mathrm{~nm}$. Enhanced microcavity emission matches the 'delayed' regime, in which contributions from triplet-triplet annihilation are significant. (e) Comparison of encapsulated (solid) and oxygen-exposed (open) films and microcavities reveals nearly identical dynamics in the prompt decay regime. The polariton-induced enhancement on long timescales disappears in the $\mathrm{O}_{2}$-exposed microcavity, confirming it arises from triplet excitons. Encapsulated microcavity kinetic is reproduced from panel $\mathrm{d}$.

absorption appears to be in the weak/intermediate-coupling regime. Similar to the diphenylanthracene cavities, we attribute the anti-crossing states to polariton branches, and emission is again entirely from the lower polariton branch. Comparison of the film and microcavity emission kinetics in Fig. 3d reveals that the prompt fluorescence dynamics remain unchanged. However, delayed fluorescence from triplet-triplet annihilation once again exhibits a longer lifetime in the microcavity. By contrast, in a reference material INDB in which we observe no contribution to emission in the bare film from triplet-triplet annihilation, we also observe no enhancement of long-lived emission in microcavities (ESI, Fig. S3 and S4:). Likewise, when we quench the triplets in DPPT through exposure to oxygen, ${ }^{50}$ the polariton-induced enhancement observed in Fig. 3d disappears (Fig. 3e; encapsulated microcavity data is reproduced from panel d).

In short, we observe that the lifetime of delayed emission can be increased through strong light-matter coupling to form exciton-polaritons. This process is directly correlated with the triplet population, and if these dark, uncoupled triplet states are quenched or absent there is no enhancement. We attribute this behaviour to population of polariton states via triplet-triplet annihilation from a reservoir of dark, uncoupled triplet excitons, but there must be an additional factor to provide a longer lifetime than observed in thin films. We propose that this effect arises from a distinct longer-lived, non-emissive state that can transfer population to the polariton, namely the high-spin triplet-pair states that arise from the encounter of two spin- 1 triplets (see Discussion below). Detailed analysis of the enhancement in diphenylanthracene and DPPT microcavities is complicated by the bimolecular nature of triplet-triplet annihilation: it also depends on (non-uniform) morphology, exciton diffusion and excitation density. We thus turn instead to a system capable of the reverse process, singlet fission, in which a single photon can generate both of the necessary triplets, which then undergo geminate triplet-triplet annihilation. ${ }^{28,45,52-54}$

\section{Singlet fission and quintet harvesting in microcavities}

We use polycrystalline films of TIPS-tetracene (Fig. 4a), which has been thoroughly characterised with a range of complementary spectroscopic techniques (optically detected magnetic resonance, transient and/or magnetic field-dependent -absorption, -photoluminescence and -EPR). ${ }^{54-57}$ These measurements have 
a

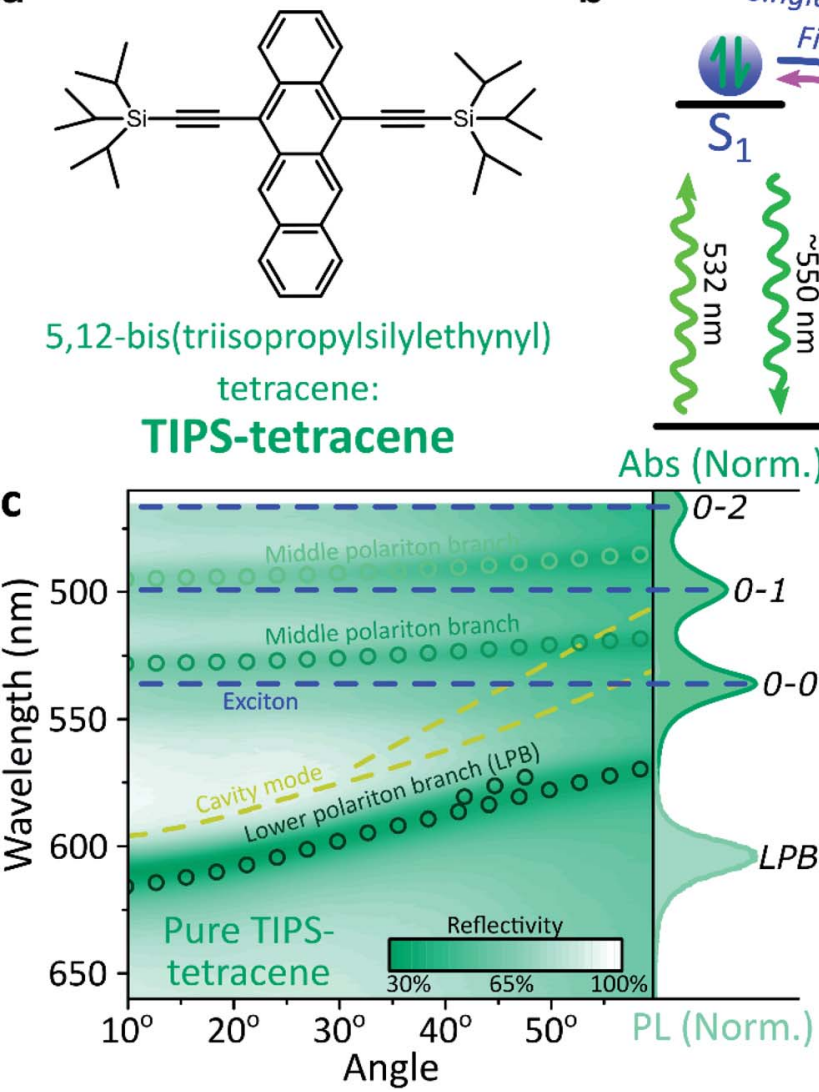

b

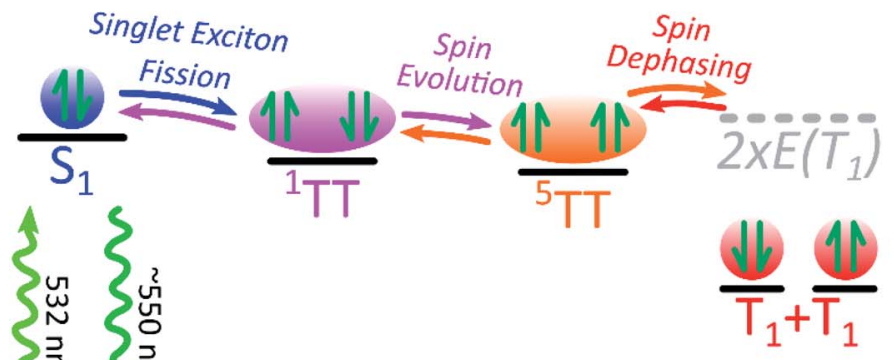

Fig. 4 Singlet fission into bound TT within a microcavity. (a) Molecular structure of TIPS-tetracene. (b) Simplified schematic of TIPS-tetracene photophysics, details in main text. All processes are potentially reversible, leading to delayed fluorescence from triplet-triplet annihilation. (c) Reflectivity map of a pure TIPS-tetracene film within Ag-Ag microcavity. Comparison with absorption spectrum (right) and transfer matrix modelling (lines, circles) confirms strong coupling to multiple vibronic transitions. Emission is from the lower polariton branch (LPB). Emission is collected with a NA $=0.76$ lens and thus effectively integrates along the entire dispersion $\left( \pm 45^{\circ}\right)$. (d) Integrated photoluminescence kinetics over full emission band for bare film (light) and microcavity (dark) following excitation at $532 \mathrm{~nm}$. All emission on these timescales arises from triplettriplet annihilation.

established a detailed picture of the excited-state and spindependent dynamics, which we directly rely on here. Rapid singlet fission from $\mathrm{S}_{1}$ generates the spin-0 triplet-pair state ${ }^{1}(\mathrm{TT})$. This can then evolve into higher-spin pair states such as ${ }^{5}(\mathrm{TT}){ }^{56,58-60}$ as well as a mixed-spin state $\left.{ }^{1}(\mathrm{TT})-^{5}(\mathrm{TT})\right)^{55}$ on sub- $\mu \mathrm{S}$ timescales. Unlike the bimolecular triplet-triplet annihilation above, this evolution does not depend on exciton diffusion, allowing us to temporally isolate the contribution of high-spin states in microcavities.

Just as in the photon up-conversion blend and DPPT, we consider the photophysical dynamics of the thin film to be active also within the exciton reservoir of our microcavities, and we outline the basic progression in TIPS-tetracene here (Fig. 4b). In these films, singlet fission occurs within $50 \mathrm{ps},{ }^{54}$ well within our instrument response of $4 \mathrm{~ns}$. The singlet and ${ }^{1}$ (TT) states are very similar in energy resulting in a dynamic equilibrium and weak delayed fluorescence. Over time, this bound triplet-pair state adopts increasing ${ }^{5}$ (TT) character ${ }^{56}$ and the films become non-emissive. This explains the fact that while the total triplet-pair population, probed with transient absorption spectroscopy (Fig. 5, solid line), does not decay significantly over $\mu$ s timescales, the delayed fluorescence (Fig. 5, open circles) does. ${ }^{54}$ It also explains the presence of ${ }^{5}$ (TT) on microsecond timescales, probed with time-resolved electron paramagnetic resonance (Fig. 5, dashed line). ${ }^{56}$ This process probably relies on off-diagonal dynamic disorder or triplethopping that promotes fluctuations in the spin-exchange coupling, ${ }^{61-64}$ see discussion below. On very long timescales $(>\mu \mathrm{s})$, spin dephasing yields pairs of triplets with independent spins that nonetheless remain bound. ${ }^{54,56}$ Due to this binding, the predominant triplet-triplet annihilation processes in TIPStetracene are geminate on all timescales, ${ }^{54}$ meaning annihilation occurs between triplets formed from the same parent singlet state and the same absorbed photon. As a result, the normalised delayed fluorescence kinetics show no dependence on excitation density allowing straightforward direct comparison of microcavity and film dynamics.

Within microcavities we observe strong coupling throughout the TIPS-tetracene absorption band and clear polariton branches, Fig. 4c. As above, emission is predominantly from the bottom of the lower polariton branch independent of the energy offset between $S_{1}$ and the cavity photon at $0^{\circ}$, the 'detuning'. 

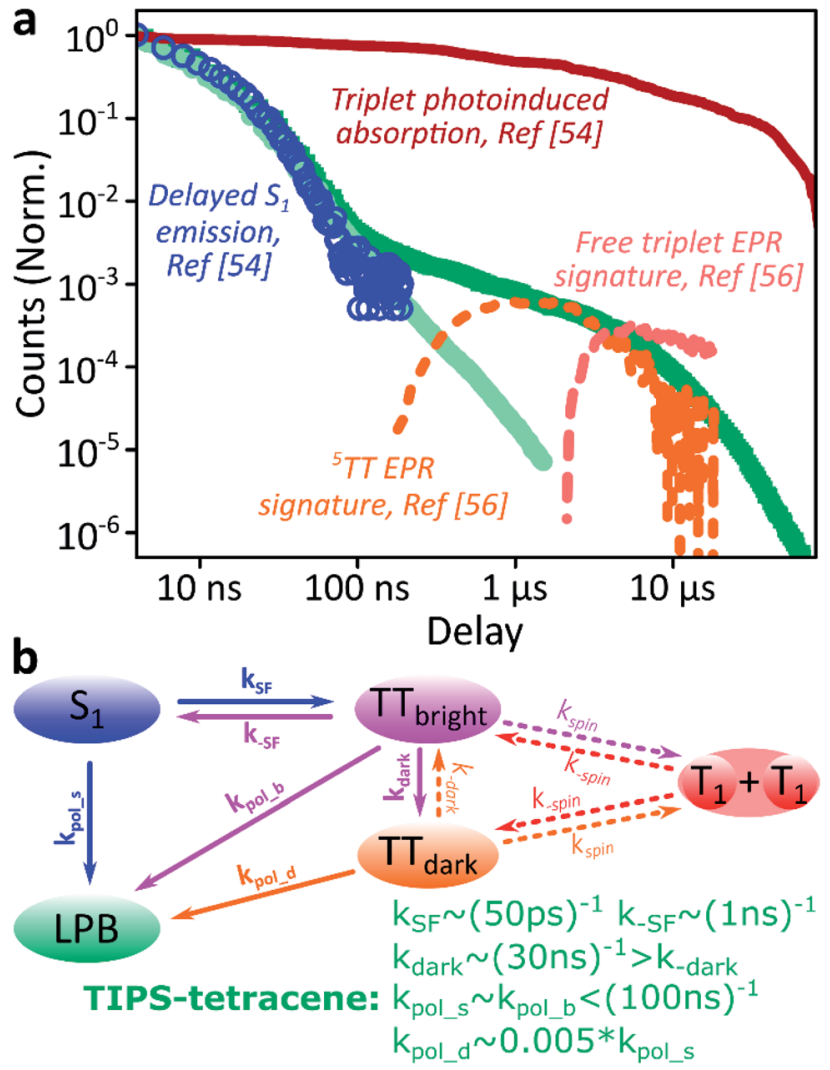

Fig. 5 Identification of the ${ }^{5} T T$ contribution. (a) Reproduction of the TIPS-tetracene film and microcavity emission kinetics from Fig. 4d, compared with time-correlated single photon counting (circles, ref. 54), transient absorption (solid, ref. 54) and electron paramagnetic resonance (dashed, ref. 56) kinetics previously reported for equivalent polycrystalline films. The long-time enhancement in microcavities coincides with observations of high-spin ${ }^{5}(T T)$. (b) Rate model used to describe dynamics within the exciton reservoir. $\mathrm{TT}_{\text {bright }}$ are triplet-pair states that directly mix with $S_{1}$. $\prod_{\text {dark }}$ states are unable to efficiently repopulate $S_{1}$. Rates shown are from fitting to published data. A similar model would describe diphenylanthracene: PtOEP or DPPT, replacing $k_{\mathrm{SF}}$ with $k_{\mathrm{ISC}}$ directly to the (single) triplet states and including the effects of exciton diffusion within $k_{\text {spin. }}$. Nonradiative decay (from all states) and radiative decay (from $\mathrm{S}_{1}, \mathrm{LPB}$ ) omitted for clarity.

Relative to the bare film, the pre-100 ns microcavity emission is only weakly perturbed, Fig. 4d. However, beyond $100 \mathrm{~ns}$ the microcavity shows a significantly enhanced long-lived tail.

This effect is qualitatively reproduced for a wide range of exciton-photon detunings, though the magnitude of enhancement varies between microcavities (ESI, Fig. S9), and the polariton population distribution along the LPB points to the existence of multiple broad energetic resonances (ESI, Fig. S10 ). We observe the same behaviour in samples where the TIPS-tetracene active layer is physically separated from the Ag mirrors with $20 \mathrm{~nm}$ spacer layers (ESI, $\$$ Section 4.2). This effect cannot be induced through any of the individual sample fabrication steps (ESI, Section 4.1) but only occurs in full strongly coupled structures. As in previous systems, the film and microcavity spectral shapes exhibit negligible evolution over this decay (ESI, Fig. S11 $)$. In the film this is because all emission we detect is from $\mathrm{S}_{1}$, populated by triplet-triplet annihilation from ${ }^{1}(\mathrm{TT})$.
Likewise, in the microcavity the constant spectral shape indicates that the emission is mediated by the lower polariton state, also populated by annihilation of triplet pairs in the reservoir. However, the clear delineation into two kinetic regimes in Fig. 4d suggests that on long times ( $>100 \mathrm{~ns}$ ) this process follows a distinct pathway that is unavailable in the film. To identify this pathway, in Fig. 5a we compare our results with published data from TIPS-tetracene polycrystalline films that show identical absorption spectra and fluorescence lifetimes to our own and are thus equivalent. ${ }^{54,56}$ Interestingly, the bulk of our microcavity-enhanced emission coincides with the time frame when quintet states are observed in the film (dashed line), and we conclude that the lower polariton can be directly populated by the quintet pairs.

We can describe the effect of strong coupling with the simple kinetic model illustrated in Fig. 5b (see ESI, $\$$ Section 5, for details). Here, $\mathrm{TT}_{\text {bright }}$ denotes well-defined triplet-pair states able to couple directly to $\mathrm{S}_{1}\left(\right.$ e.g. $\left.{ }^{1}(\mathrm{TT})\right)$; these states quickly establish a thermal equilibrium with $S_{1}$ upon which spin evolution of the pair occurs. ${ }^{54} \mathrm{TT}_{\text {dark }}$ includes all pair states that do not directly or strongly interact with $\mathrm{S}_{1}$, such as the previously observed ${ }^{5}$ (TT) state and the mixed-spin ${ }^{1}(\mathrm{TT})-^{5}(\mathrm{TT})$ state. Using published data ${ }^{54,56,65}$ to fix the forward and reverse singlet fission rates $k_{(-) \mathrm{SF}}$, the conversion rates between bright and dark TT states $k_{(-) \text {dark }}$ and the rate of spin dephasing into the uncoupled pair $\left(\mathrm{T}_{1}+\mathrm{T}_{1}\right) k_{(-) \text {spin, }}$ we arrive at a highly constrained model that can simultaneously describe all reported neat-film TIPS-tetracene dynamics, ${ }^{54-56}$ including our own data. To describe the situation in our microcavities, we include the new emissive LPB state and use the same intrinsic rate constants for the photophysics involving states within the exciton reservoir. The only new rate constants that appear, $k_{\text {pol_x }}$, describe the transfer of population from the exciton reservoir states $\mathrm{x}$ to the $\mathrm{LPB}$. We have identified only two scenarios that agree with our experimental results for the microcavity. (1) The rate constant $k_{- \text {dark }}$ is greatly enhanced, enabling harvesting of the large $\mathrm{TT}_{\text {dark }}$ population via $\mathrm{S}_{1}$, while $k_{\text {pol_d }}$ is negligible. The LPB in this regime is only directly populated from relaxation of singlet states in the reservoir. This would mean that strong coupling to $\mathrm{S}_{1}$ perturbs the spin physics of uncoupled states within the cavity. Alternatively, (2) $k_{\text {pol_d }}>$ 0 and all other rates remain the same, allowing direct transfer from the reservoir of $\mathrm{TT}_{\mathrm{dark}}$ excitons into the LPB. While both routes are very surprising, we consider the $k_{\text {pol_d }}$ pathway to be much more probable, and it provides a better fit to the full dataset (ESI, Fig. S15 ). The chief effect of strong coupling is thus to open an energetically downhill pathway for population transfer from $\mathrm{TT}_{\text {dark }}$ into the emissive LPB. Let us briefly abound in this latter proposed scenario.

It is interesting that given the long lifetime of the $\mathrm{TT}_{\text {dark }}$ excitons and the fast cavity leakage rate, the $\mathrm{TT}_{\text {dark }} \rightarrow$ LPB rate does not need to be large to enable a large effect in the delayed photoluminescence. The associated downhill population transfer can occur via a combination of radiative pumping or via nonadiabatic transfer mechanisms, where the rate-limiting step is respectively mediated by photonic or matter components. In radiative pumping, a small $\mathrm{S}_{1}$ fraction of the $\mathrm{TT}_{\text {dark }}$ manifold 
allows for the emission of phonons and a red-shifted virtual photon $^{52}$ that is absorbed by the LPB; in nonadiabatic transfer, the electronic coupling between $\mathrm{TT}_{\text {dark }}$ and LPB mediates the transfer of population accompanied by emission of phonons. ${ }^{\mathbf{4 1}}$ In either mechanism, there needs to be a finite electronic coupling between $\mathrm{TT}_{\text {dark }}$ to $\mathrm{S}_{1}$ which, although expected to be very small, is large enough to provide the physical mechanism whereby the $\mathrm{TT}_{\text {dark }}$ reservoir can directly feed the LPB.

\section{Discussion}

In all the systems we have studied in which triplets are formed, triplet-triplet annihilation leads to longer-lived emission in the strong-coupled microcavity compared with the bare film. Because of the long timescales involved, this is a significant effect even though the prompt emission from these states is weak. Integration of the kinetics in Fig. 2-4 suggests a polaritoninduced enhancement to the total emission of up to $150 \%$ (ESI, Fig. S16 ). Based on the model system TIPS-tetracene, and the fact that in all systems in which we observe microcavityenhanced emission on these timescales the primary species are uncoupled triplet excitons, we have suggested that the enhanced emission comes from harvesting ${ }^{5}$ (TT) into the lower polariton. This result is entirely unprecedented: direct interaction between these pair-states and the polariton is spin- and symmetry-forbidden, and thus very weak. Thus, our observations show that strong coupling can alter the photophysics even of states that cannot interact directly with light.

\section{Theoretical foundations of the kinetic model}

To shed more insight into the experimental results above, we highlight the essential features of the kinetic model (see Fig. 6a, which is a simplified version of Fig. 5 b). As emphasized before, we consider rate constants of transfer between dark exciton manifolds as identical to the corresponding rate constants in the cavity-free case. We also expect the rates that account for transfer from dark exciton reservoirs to polariton modes $\left(k_{\text {pol_x }}\right)$ to be smaller than their corresponding out-of-cavity analogues (e.g. population transfer between $\mathrm{TT}_{\text {dark }}$ and $\mathrm{S}_{1}$ ) by $N_{\text {eff }} \approx$ $\mathrm{O}\left(10^{6}\right)-\mathrm{O}\left(10^{9}\right)$ to account for the deleterious effect of delocalization of the final polariton states. ${ }^{40}$ This dilution factor can be intuitively explained through the mismatch in wavefunction delocalization of these states: in the presence of disorder, the dark excitons can be regarded as essentially localized on particular molecular sites while polaritons are delocalized through the coherence length of the optical cavity mode, so the overlap between initial and final states scales as $1 / N_{\text {eff. }}$. These 'direct' processes between dark states and the polariton modes may thus seem strongly disfavoured but, as we shall argue, they play a crucial role in the long-time photophysics of the presently studied organic polariton systems.

Another important fact to consider is that singlet fission in TIPS-tetracene occurs on the picosecond timescale, ${ }^{54}$ whereas the triplet-triplet annihilation process lifetime is on the order of nanoseconds, ${ }^{65}$ the latter being approximately one order of magnitude faster than the fluorescence lifetime of the bright singlet excitons (see Fig. 6a and ESI for the rate constants used in our model and their justification). In the bare film scenario, these conditions imply that a thermal equilibrium which is heavily biased towards the exciton reservoir of $\mathrm{TT}_{\text {bright }}$ states is quickly established well before emission of a photon occurs via the $S_{1}$ states. The additional irreversible transfer from $\mathrm{TT}_{\text {bright }}$ to $\mathrm{TT}_{\text {dark }}$ states leads to the accumulation of population in the latter. Once most of the initial population is trapped in the $\mathrm{TT}_{\text {dark }}$ manifold, the fluorescence signal is negligible as a result of the slow back transfer from $\mathrm{TT}_{\text {dark }}$ to $\mathrm{TT}_{\text {bright }}$ states, and the presumably small (effective) coupling between $\mathrm{TT}_{\text {dark }}$ and $\mathrm{S}_{1}$
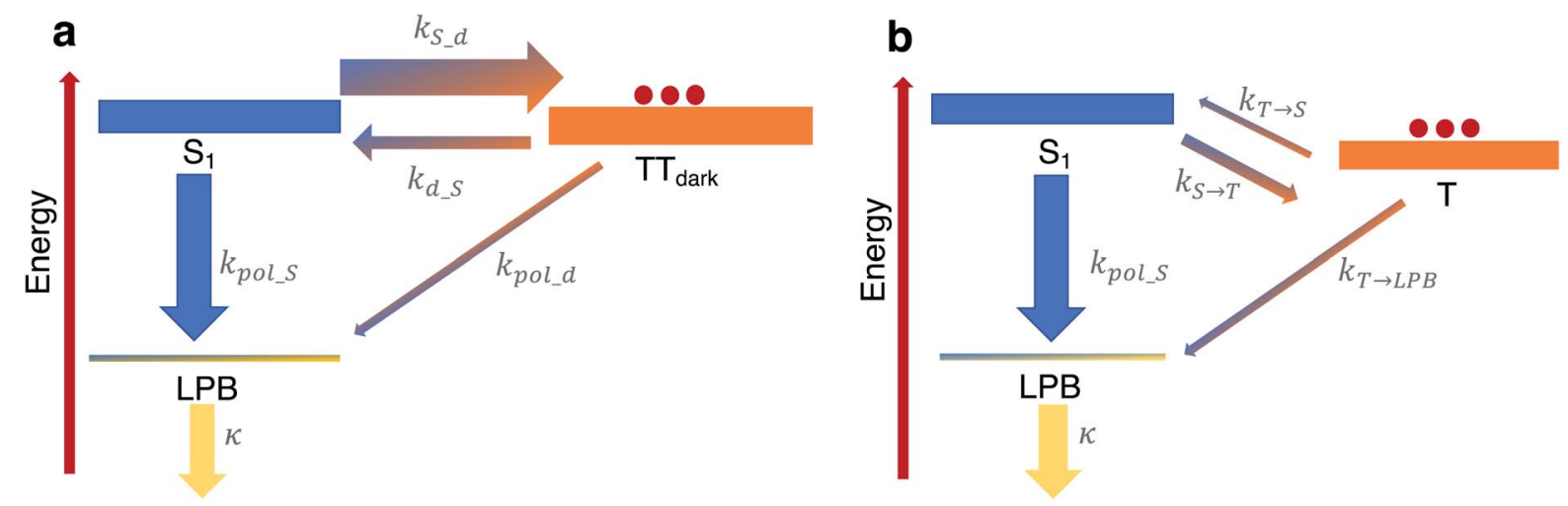

Fig. 6 Relevant relaxation pathways within the microcavity. The significant difference between the density of states of the (dark) exciton reservoirs (thick rectangles) and the polariton branches (thin rectangles) renders the transitions between the exciton reservoirs (thick arrows) faster than transfer of population from a reservoir to polariton modes (thin arrows). (a) In TIPS-tetracene, we argue that a large rate constant $k_{S_{-}} d$ relative to the corresponding one for the inverse process $k_{\text {d_s }_{1}} \ll k_{\mathrm{s}_{-} d}$ effectively traps the equilibrated population in the $T T_{\text {dark }}$ manifold. The most efficient channel of photon emission would be through the direct transfer to the LPB, in spite of $k_{d_{-} s} \gg k_{\text {pol_d }}$, since the leakage rate $k \gg k_{\text {pol_d }}$ releases photons out of the cavity very quickly. For simplicity, our scheme here omits the $\Pi_{\text {bright }}$ manifold (see Fig. $5 b$ ) since we consider the long-time limit where the delayed photoluminescence is determined by the population in $T_{\text {dark. }}$ (b) In contrast to our results, in the scenario of a faster feeding of the LPB by the dark $S_{1}$ manifold compared to the timescales of transition between triplet $T$ and dark $S_{1}$ states (as in thermallyactivated delayed fluorescence chromophores), the delayed photoluminescence signal is, in general, expected to be the same in both microcavity and cavity-free scenarios, since its decay profile is determined by the rate-limiting step associated with $k_{\mathrm{T} \rightarrow \mathrm{s}}$. 
states. This behavior is captured in Fig. 6a by the coarse-grained effective rate constants $k_{\mathrm{S} \_d}$ and $k_{\mathrm{d} \_s}$, which are heavily biased towards $\mathrm{TT}_{\text {dark }}$ (these effective rate constants are implicit functions of $k_{\mathrm{SF}}, k_{-\mathrm{SF}}, k_{\text {dark }}, k_{- \text {dark }}$ and therefore, should be understood as incorporating the mediating role of $\mathrm{TT}_{\text {bright }}$, which we omit for simplicity).

While the coupling between $\mathrm{TT}_{\text {dark }}$ and $\mathrm{S}_{1}$ states is small and irrelevant in the cavity-free case, it turns out to be of prime relevance inside of the microcavity as it provides the only direct channel to funnel $\mathrm{TT}_{\text {dark }}$ population into the LPB. This process is guaranteed to be slow due to the small electronic mixing between $\mathrm{TT}_{\text {dark }}$ and $\mathrm{S}_{1}$, as well as the large dilution factor of $N_{\text {eff. }}$. Yet, it is followed by a very fast photonic leakage via the photonic component of LPB. Thus, photon emission via the direct pathway $\mathrm{TT}_{\mathrm{dark}} \rightarrow$ LPB ends up being more efficient than its $\mathrm{TT}_{\text {dark }} \rightarrow \mathrm{S}_{1} \rightarrow$ LPB counterpart, where the equilibrium of dark $\mathrm{S}_{1} \leftrightarrow \mathrm{TT}_{\text {dark }}$ (largely biased towards $\mathrm{TT}_{\text {dark }}$ ) precludes the population trapped in $\mathrm{TT}_{\text {dark }}$ to reach the dark $\mathrm{S}_{1}$ states in due time (see Fig. 6a). This biased equilibrium implies that this detrapping of $\mathrm{TT}_{\text {dark }}$ population via the LPB is expected to occur even when $\mathrm{TT}_{\text {dark }} \rightarrow \mathrm{S}_{1}$ (which is already small) is faster than $\mathrm{TT}_{\text {dark }} \rightarrow$ LPB.

Our present result seems at odds with previous reports showing that the LPB does not necessarily offer an advantage on the harvesting of delayed fluorescence from triplets in organic materials, even when the lower polariton branch is below the triplet states. ${ }^{40}$ To understand this apparent discrepancy, we notice the difference in timescales between singlet fission/triplettriplet annihilation (relevant in the present experiment) and those for reverse- and intersystem-crossing (which are the mechanisms that come into play for individual triplet harvesting; see Fig. 6b). Both reverse- and intersystem crossing processes are on the $\mu$ s timescale, ${ }^{66}$ three orders of magnitude slower than the fluorescence lifetime of singlets. Upon strong coupling, the population trapped in the dark triplet states gives rise to photoluminescence through the most efficient channel, which in this case corresponds to the $\mathrm{T} \rightarrow \mathrm{S}_{1} \rightarrow$ LPB pathway, where $\mathrm{T}$ accounts for the triplet states. The direct $\mathrm{T} \rightarrow$ LPB pathway is unable to compete with the indirect pathway in view of the large density of states of the T manifold compared to the LPB. In other words, the $\mathrm{T}$ population is not as efficiently trapped in Fig. $6 \mathrm{~b}$ as the $\mathrm{TT}_{\text {dark }}$ population is in Fig. 6a, so the direct $\mathrm{T} \rightarrow$ LPB pathways offers no incentives to be utilized. In consequence, the expected decay profile of the microcavity photoluminescence follows the same timescale as that for the cavity-free case, given that the rate-limiting step in both cavity-free and cavity cases is the very slow reverse intersystem crossing channel from triplet to singlet reservoirs. To summarize, the mechanism in Fig. $6 \mathrm{~b}$ is in sharp contrast with our results, where the predominant mechanism of photoluminescence is different in the microcavity compared to the bare film case.

\section{Role of mixed adiabatic states}

A critical element in cavity mediated harvesting of TT population in TIPS-tetracene and related systems is that there must be nonzero electrostatic coupling between the high-spin pair TT states and the polariton. The process of singlet fission is generally described in simplistic terms, where a singlet exciton splits into a pair of triplet excitons which are initially coupled into a triplet pair. In reality, these excited states are not pure diabatic states but rather mixed adiabatic states. Thus the nominal $S_{1}$ state is often substantially mixed with higher-lying charge-transfer excitons $(\mathrm{CT})^{67}$ and even attains some TT character. The ${ }^{1}$ (TT) state is also substantially mixed with $\mathrm{CT}$ and $\mathrm{S}_{1}$, resulting in significant binding with respect to two free triplets ${ }^{52,53,68-70}$ (essential for fission to even be possible in TIPS-tetracene ${ }^{54}$ ) and providing a channel for this formally dark state to emit directly. ${ }^{52-54,70}$ The mixed character of both states enables ultrafast singlet fission $^{52,54,71,72}$ and efficient equilibration between (adiabatic) $\mathrm{S}_{1}$ and ${ }^{1}(\mathrm{TT}) .{ }^{54}$ Once formed, the adiabatic ${ }^{1}(\mathrm{TT})$ state can exhibit further evolution. The constituent triplets within the pair can interact through exchange coupling. In the regime of weak

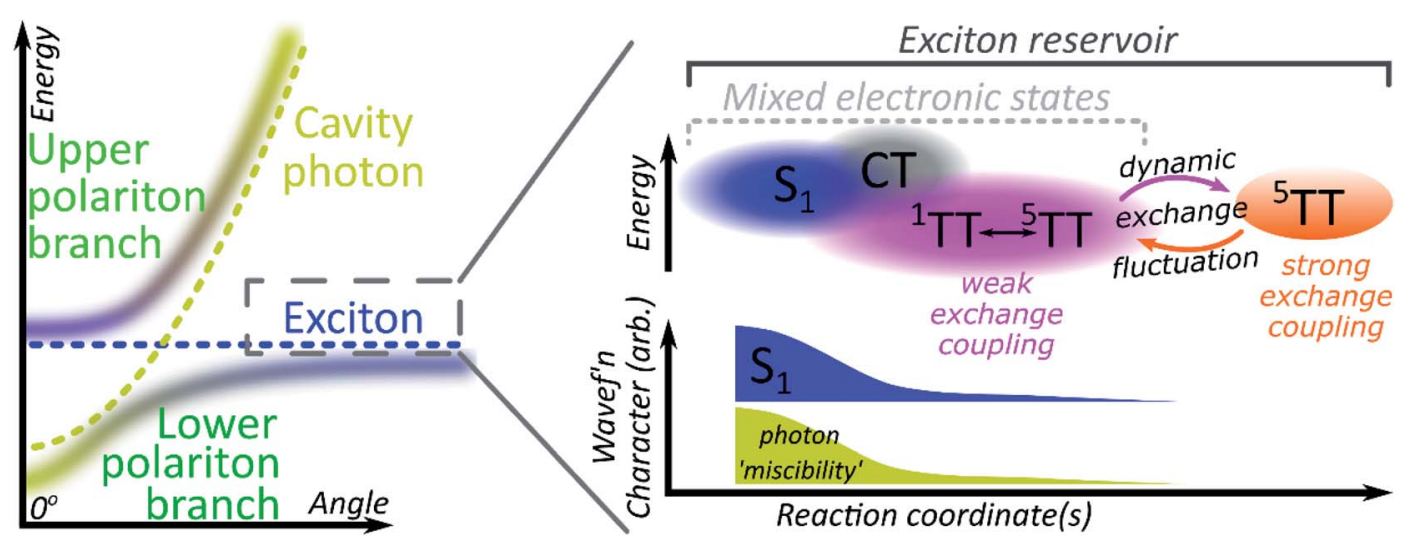

Fig. 7 Mixed electronic states within a microcavity. Illustrative schematic of the energy landscape and mixed electronic states that contribute to the reservoir of dark excitons. The latter shall be understood as composed of states with chemical character varying on a continuum along a generalised reaction coordinate, e.g. exhibiting increasing mixing with CT and TT states. While the adiabatic states are often named according to their predominant character, other states also contribute to their wavefunctions. Here, mixing with $\mathrm{S}_{1}$ allows 'miscibility' with the photon through the $\mathrm{S}_{1}$-polaritons. The coupling of the pure ${ }^{5}(\mathrm{TT})$ state to the photon is small, yet crucial to the cavity mediated harvesting of TT population demonstrated in this study. 
exchange coupling, the initially created ${ }^{1}(\mathrm{TT})$ state mixes with other spin configurations via dipolar interactions, resulting in a triplet pair with mixed singlet and quintet character. ${ }^{73}$ This regime has been detected in TIPS-tetracene using magnetic resonance techniques, ${ }^{55}$ and it is likely that the formation of this dark ${ }^{1}(\mathrm{TT})-^{5}(\mathrm{TT})$ mixed state is responsible for the $\sim 10 \mathrm{~ns}$ decay of delayed fluorescence in films ${ }^{54}$ as the state loses its (pure) singlet character. The recent observation in the same and other materials of strongly exchange-coupled triplet pairs suggests that the exchange interaction must fluctuate over time. ${ }^{56,58-60,62,74}$ The coexistence of these two regimes on similar timescales indicates that the weakly and strongly exchange-coupled triplet pairs are in dynamic equilibrium, probably driven by thermal fluctuations in the exchange interaction (off-diagonal dynamic disorder) ${ }^{61}$ Importantly, the physical mechanism behind the fluctuating exchange coupling is not linked to singlet fission but is an intrinsic property of organic materials. We can thus expect comparable spin evolution to occur in triplet-triplet annihilation systems, where the first step is formation of a TT encounter complex.

\section{Conclusions}

We see, then, that all of the relevant electronic states in the singlet fission pathway are connected by excited-state mixing, as shown in Fig. 7. Mixed adiabatic states are a critical driver in photophysical processes such as ultrafast intersystem crossing, ${ }^{75}$ thermally activated delayed fluorescence, ${ }^{76}$ singlet exciton fission ${ }^{52,54}$ and its reverse, triplet-triplet annihilation. This property is a critical distinction from inorganic semiconductors, but it is rarely considered explicitly in the excitonpolariton field. Thus, when the photon couples to $S_{1}$ to form polaritons, it in fact interacts with all the states that mix with $S_{1}$. Consequently, all states that mix with $\mathrm{S}_{1}$ may acquire some ability to interact with the polaritonic states. In polaritonic systems in which triplet-triplet annihilation occurs, this creates a pathway to populate the radiative lower polariton branch and thus a route to harvest light from nominally dark diabatic states. Whether the mechanism of polariton feeding proceeds through a radiative (photon-mediated) or a nonradiative (material-mediated) will require further research.

The matrix element coupling the dark pair-states to $S_{1}$ must exist even in the neat film, but the coupling is evidently too small to overcome the energetic or kinetic barriers against delayed emission, and very little is detected. The microcavity offers changes to the energetic structure and reorganisation energies, ${ }^{\mathbf{4 0 , 4 1}}$ as well as additional radiative and nonradiative rates (Fig. 5 and 6) that enable even this weak coupling element to contribute significantly to emission. While the above explains any microcavity emission originating from ${ }^{1}$ (TT), it does not on its own explain our observation of ${ }^{5}$ (TT) harvesting. Our cavity modified kinetics reveal a correlation with the strongly exchange-coupled, pure ${ }^{5}(\mathrm{TT})$ state. The latter exists in equilibrium with the weakly exchange-coupled ${ }^{1}(\mathrm{TT})-^{5}(\mathrm{TT})$ mixed state, ${ }^{55,56,61,73}$ which itself can mix with the photon through its ${ }^{1}$ (TT) component. Hence, in the strong excitonphoton coupling regime, mixed states gain a weak but non-zero coupling element to populate the emissive lower polariton branch. Thanks to dynamic fluctuations in the exchange coupling, this allows even completely dark states like ${ }^{5}(\mathrm{TT})$ to serve as a reservoir for polariton emission.

Interestingly, this behaviour could allow for an improvement in solid-state photon up-conversion. Simple spin considerations suggest that the maximum quantum efficiency of up-conversion should be $\sim 11 \%$, as only 1 of 9 possible TT pair states/ encounter complexes have zero spin (i.e. ${ }^{1}(\mathrm{TT})$ ). Distinctly higher efficiencies have been observed in solution, attributed to the higher spin-states such as ${ }^{5}$ (TT) dissociating without significant loss to reform ${ }^{1}$ (TT). ${ }^{46,77}$ This has never been observed in the solid state. In an optimised fluorescence up-conversion system, the ability to directly harvest ${ }^{5}$ (TT) encounter complexes in the weak spin-interaction regime should boost the maximum efficiency. At the same time, the resulting up-converted polariton emission would be well-directed, thereby simplifying collection. A similar mechanism could be used in electrically injected polariton LEDs and lasers, where triplet excitons constitute $75 \%$ of the population and triplet-triplet annihilation could be used to harvest them. Because these states are very long-lived, they can make a substantial contribution to the total emission even if the instantaneous probability to emit is always low. ${ }^{78}$ The ability of these very longlived states to populate the lower polariton can also enable new applications in polaritonic physics. For example, it may be possible to use such a reservoir of non-coupled states to feed a polariton condensate, increasing its effective lifetime. This may be equivalent to the continuous pumping of exciton reservoir states in GaAs microcavities to continually repopulate the polariton condensate. ${ }^{13}$ Such long-lived condensates would be important for practical applications of room-temperature polariton lasing. This concept also vastly expands the scope of microcavity-controlled chemistry, which seeks to alter material properties and light-induced dynamics through strong light-matter coupling. ${ }^{\mathbf{1 - 3 , 6 - 1 1}}$ The interactions implicit in the adiabatic picture mean that strong coupling may perturb not only the state that dominates the absorption spectrum, but also some states that mix with it. Our work presents new opportunities to control processes in which an absorbing state mixes with dark states, for example charge transfer, biological light harvesting, energy transfer and intersystem crossing.

\section{Author contributions}

DWP, HC, TPL and AJM performed the optical experiments. RJ performed transfer-matrix modelling. LAMM and JYZ performed theoretical analysis. AL, KJF, AJP, JA and HB synthesised materials. DWP, DGB and KG prepared samples. DWP, JC and AJM analysed the data and wrote the manuscript. AJM conceived the project and supervised it with JC. All authors discussed the results and commented on the manuscript.

\section{Conflicts of interest}

There are no conflicts to declare.

\section{Acknowledgements}

This work was supported by the Engineering and Physical Sciences Research Council, U.K. (Grant Number EP/M025330/1, 
'Hybrid Polaritonics'), the University of Sheffield and EU project 679789-455 'CONTREX'. DGB was supported by the EPSRC Centre for Doctoral Training in New and Sustainable Photovoltaics (EP/L01551X/1). TL and AIT thank the financial support of the European Graphene Flagship Project under grant agreement 785219 and EPSRC grant EP/P026850/1. LAMM and JYZ were supported by the US Department of Energy, Office of Science, Basic Energy Sciences, CPIMS Program under Early Career Research Program award DE-SC0019188. AJM gratefully acknowledges LR Weiss for useful discussion about exchange coupling. The spectroscopic work was performed at the EPSRC Lord Porter Laser Facility, EP/L022613/1 and EP/R042802/1.

\section{Notes and references}

1 J. A. Hutchison, T. Schwartz, C. Genet, E. Devaux and T. W. Ebbesen, Angew. Chem., Int. Ed., 2012, 51, 1592-1596.

2 D. M. Coles, N. Somaschi, P. Michetti, C. Clark, P. G. Lagoudakis, P. G. Savvidis and D. G. Lidzey, Nat. Mater., 2014, 13, 712-719.

3 E. Orgiu, J. George, J. A. Hutchison, E. Devaux, J. F. Dayen, B. Doudin, F. Stellacci, C. Genet, J. Schachenmayer, C. Genes, G. Pupillo, P. Samorì and T. W. Ebbesen, Nat. Mater., 2015, 14, 1123-1130.

4 A. Shalabney, J. George, J. Hutchison, G. Pupillo, C. Genet and T. W. Ebbesen, Nat. Commun., 2015, 6, 5981.

5 J. P. Long and B. S. Simpkins, ACS Photonics, 2015, 2, 130136.

6 A. Thomas, L. Lethuillier-Karl, K. Nagarajan, R. M. A. Vergauwe, J. George, T. Chervy, A. Shalabney, E. Devaux, C. Genet, J. Moran and T. W. Ebbesen, Science, 2019, 363, 615-619.

7 F. Herrera and F. C. Spano, Phys. Rev. Lett., 2016, 116, 238301.

8 T. W. Ebbesen, Acc. Chem. Res., 2016, 49, 2403-2412.

9 L. A. Martínez-Martínez, M. Du, R. F. Ribeiro, S. Kéna-Cohen and J. Yuen-Zhou, J. Phys. Chem. Lett., 2018, 9, 1951-1957.

10 J. Flick, M. Ruggenthaler, H. Appel and A. Rubio, Proc. Natl. Acad. Sci. U. S. A., 2017, 114, 3026-3034.

11 J. Feist, J. Galego and F. J. Garcia-Vidal, ACS Photonics, 2018, 5, 205-216.

12 J. Kasprzak, M. Richard, S. Kundermann, A. Baas, P. Jeambrun, J. M. J. Keeling, F. M. Marchetti, M. H. Szymańska, R. André, J. L. Staehli, V. Savona, P. B. Littlewood, B. Deveaud and L. S. Dang, Nature, 2006, 443, 409-414.

13 A. Amo, D. Sanvitto, F. P. Laussy, D. Ballarini, E. del Valle, M. D. Martin, A. Lemaître, J. Bloch, D. N. Krizhanovskii, M. S. Skolnick, C. Tejedor and L. Viña, Nature, 2009, 457, 291-295.

14 K. S. Daskalakis, S. A. Maier, R. Murray and S. Kena-Cohen, Nat. Mater., 2014, 13, 271-278.

15 G. Lerario, A. Fieramosca, F. Barachati, D. Ballarini, K. S. Daskalakis, L. Dominici, M. De Giorgi, S. A. Maier, G. Gigli, S. Kéna-Cohen and D. Sanvitto, Nat. Phys., 2017, 13, 837-841.
16 N. G. Berloff, M. Silva, K. Kalinin, A. Askitopoulos, J. D. Töpfer, P. Cilibrizzi, W. Langbein and P. G. Lagoudakis, Nat. Mater., 2017, 16, 1120-1126.

17 D. Sanvitto and S. Kéna-Cohen, Nat. Mater., 2016, 15, 10611073.

18 C. Schneider, A. Rahimi-Iman, N. Y. Kim, J. Fischer, I. G. Savenko, M. Amthor, M. Lermer, A. Wolf, L. Worschech, V. D. Kulakovskii, I. A. Shelykh, M. Kamp, S. Reitzenstein, A. Forchel, Y. Yamamoto and S. Höfling, Nature, 2013, 497, 348-352.

19 A. Graf, M. Held, Y. Zakharko, L. Tropf, M. C. Gather and J. Zaumseil, Nat. Mater., 2017, 16, 911-917.

20 M. Held, A. Graf, Y. Zakharko, P. Chao, L. Tropf, M. C. Gather and J. Zaumseil, Adv. Opt. Mater., 2018, 6, 1700962.

21 E. Eizner, J. Brodeur, F. Barachati, A. Sridharan, S. KénaCohen, A. Sridharan and S. Kéna-Cohen, ACS Photonics, 2018, 5, 2921-2927.

22 D. G. Lidzey, D. D. C. Bradley, M. S. Skolnick, T. Virgili, S. Walker and D. M. Whittaker, Nature, 1998, 395, 53-55.

23 J. George, S. Wang, T. Chervy, A. Canaguier-Durand, G. Schaeffer, J.-M. Lehn, J. A. Hutchison, C. Genet and T. W. Ebbesen, Faraday Discuss., 2015, 178, 281-294.

24 F. Barachati, J. Simon, Y. A. Getmanenko, S. Barlow, S. R. Marder and S. Kéna-Cohen, ACS Photonics, 2018, 5, 119-125.

25 R. J. Holmes and S. R. Forrest, Phys. Rev. B: Condens. Matter Mater. Phys., 2005, 71, 235203.

26 J. D. Plumhof, T. Stoeferle, L. Mai, U. Scherf and R. F. Mahrt, Nat. Mater., 2014, 13, 247-252.

27 T. Cookson, K. Georgiou, A. Zasedatelev, R. T. Grant, T. Virgili, M. Cavazzini, F. Galeotti, C. Clark, N. G. Berloff, D. G. Lidzey and P. G. Lagoudakis, Adv. Opt. Mater., 2017, 5, 1700203.

28 C. J. Bardeen, MRS Bull., 2013, 38, 65-71.

29 D. M. Coles, P. Michetti, C. Clark, W. C. Tsoi, A. M. Adawi, J. Kim and D. G. Lidzey, Adv. Funct. Mater., 2011, 21, 36913696.

30 S. Kéna-Cohen and S. R. Forrest, Nat. Photonics, 2010, 4, 371375.

31 D. Ballarini, M. De Giorgi, S. Gambino, G. Lerario, M. Mazzeo, A. Genco, G. Accorsi, C. Giansante, S. Colella, S. D'Agostino, P. Cazzato, D. Sanvitto and G. Gigli, Adv. Opt. Mater., 2014, 2, 1076-1081.

32 R. T. Grant, P. Michetti, A. J. Musser, P. Gregoire, T. Virgili, E. Vella, M. Cavazzini, K. Georgiou, F. Galeotti, C. Clark, J. Clark, C. Silva and D. G. Lidzey, Adv. Opt. Mater., 2016, 4, 1615-1623.

33 A. J. Musser, S. K. Rajendran, K. Georgiou, L. Gai, R. T. Grant, Z. Shen, M. Cavazzini, A. Ruseckas, G. A. Turnbull, I. D. W. Samuel, J. Clark and D. G. Lidzey, J. Mater. Chem. C, 2017, 5, 8380-8389.

34 M. Du, L. A. Martínez-Martínez, R. F. Ribeiro, Z. Hu, V. M. Menon and J. Yuen-Zhou, Chem. Sci., 2018, 9, 6659-6669.

35 A. Köhler and H. Bässler, Mater. Sci. Eng., R, 2009, 66, 71109.

36 M. A. Baldo, D. F. O'Brien, Y. You, A. Shoustikov, S. Sibley, M. E. Thompson and S. R. Forrest, Nature, 1998, 395, 151154. 
37 D. N. Congreve, J. Lee, N. J. Thompson, E. Hontz, S. R. Yost, P. D. Reusswig, M. E. Bahlke, S. Reineke, T. Van Voorhis and M. A. Baldo, Science, 2013, 340, 334-337.

38 A. Rao, P. C. Y. Chow, S. Gélinas, C. W. Schlenker, C.-Z. Li, H.-L. Yip, A. K. Y. Jen, D. S. Ginger and R. H. Friend, Nature, 2013, 500, 435-439.

39 K. Stranius, M. Hertzog and K. Börjesson, Nat. Commun., 2018, 9, 2273.

40 E. Eizner, L. A. Martínez-Martínez, J. Yuen-Zhou and S. KénaCohen, arXiv 1903.09251, 2019, 1-29.

41 L. A. Martínez-Martínez, E. Eizner, S. Kéna-Cohen and J. Yuen-Zhou, J. Chem. Phys., 2019, 151, 054106.

42 A. M. Berghuis, A. Halpin, Q. Le-Van, M. Ramezani, S. Wang, S. Murai and J. Gómez Rivas, Adv. Funct. Mater., 2019, 1901317.

43 R. E. Merrifield, P. Avakian and R. P. Groff, Chem. Phys. Lett., 1969, 3, 155-157.

44 J. J. Burdett and C. J. Bardeen, J. Am. Chem. Soc., 2012, 134, 8597-8607.

45 M. B. Smith and J. Michl, Chem. Rev., 2010, 110, 6891-6936. 46 T. N. Singh-Rachford and F. N. Castellano, Coord. Chem. Rev., 2010, 254, 2560-2573.

47 R. Karpicz, S. Puzinas, V. Gulbinas, A. Vakhnin, A. Kadashchuk and B. P. Rand, Chem. Phys., 2014, 429, 5762.

48 R. R. Islangulov, J. Lott, C. Weder and F. N. Castellano, J. Am. Chem. Soc., 2007, 129, 12652-12653.

49 Y. Y. Cheng, T. Khoury, R. G. C. R. Clady, M. J. Y. Tayebjee, N. J. Ekins-Daukes, M. J. Crossley and T. W. Schmidt, Phys. Chem. Chem. Phys., 2010, 12, 66-71.

50 C. Schweitzer and R. Schmidt, Chem. Rev., 2003, 103, 16851757.

51 E. Salvadori, N. Luke, J. Shaikh, A. Leventis, H. Bronstein, C. W. M. Kay and T. M. Clarke, J. Mater. Chem. A, 2017, 5, 24335-24343.

52 C. K. Yong, A. J. Musser, S. L. Bayliss, S. Lukman, H. Tamura, O. Bubnova, R. K. Hallani, A. Meneau, R. Resel, M. Maruyama, S. Hotta, L. M. Herz, D. Beljonne, J. E. Anthony, J. Clark and H. Sirringhaus, Nat. Commun., 2017, 8, 15953.

53 S. Lukman, J. M. Richter, L. Yang, P. Hu, J. Wu, N. C. Greenham and A. J. Musser, J. Am. Chem. Soc., 2017, 139, 18376-18385.

54 H. L. Stern, A. Cheminal, S. R. Yost, K. Broch, S. L. Bayliss, K. Chen, M. Tabachnyk, K. Thorley, N. Greenham, J. M. Hodgkiss, J. Anthony, M. Head-Gordon, A. J. Musser, A. Rao and R. H. Friend, Nat. Chem., 2017, 9, 1205-1212.

55 S. L. Bayliss, A. D. Chepelianskii, A. Sepe, B. J. Walker, B. Ehrler, M. J. Bruzek, J. E. Anthony and N. C. Greenham, Phys. Rev. Lett., 2014, 112, 238701.

56 L. R. Weiss, S. L. Bayliss, F. Kraffert, K. J. Thorley, J. E. Anthony, R. Bittl, R. H. Friend, A. Rao, N. C. Greenham and J. Behrends, Nat. Phys., 2017, 13, 176181.

57 S. L. Bayliss, L. R. Weiss, A. Mitioglu, K. Galkowski, Z. Yang, K. Yunusova, A. Surrente, K. J. Thorley, J. Behrends, R. Bittl,
J. E. Anthony, A. Rao, R. H. Friend, P. Plochocka, P. C. M. Christianen, N. C. Greenham and A. D. Chepelianskii, Proc. Natl. Acad. Sci. U. S. A., 2018, 115, 5077-5082.

58 M. J. Y. Tayebjee, S. N. Sanders, E. Kumarasamy, L. M. Campos, M. Y. Sfeir and D. R. McCamey, Nat. Phys, 2017, 13, 182-188.

59 B. S. Basel, J. Zirzlmeier, C. Hetzer, B. T. Phelan, M. D. Krzyaniak, S. R. Reddy, P. B. Coto, N. E. Horwitz, R. M. Young, F. J. White, F. Hampel, T. Clark, M. Thoss, R. R. Tykwinski, M. R. Wasielewski and D. M. Guldi, Nat. Commun., 2017, 8, 15171.

60 D. Lubert-Perquel, E. Salvadori, M. Dyson, P. N. Stavrinou, R. Montis, H. Nagashima, Y. Kobori, S. Heutz and C. W. M. Kay, Nat. Commun., 2018, 9, 4222.

61 J. Aragó and A. Troisi, Phys. Rev. Lett., 2015, 114, 026402.

62 M. Wakasa, M. Kaise, T. Yago, R. Katoh, Y. Wakikawa and

T. Ikoma, J. Phys. Chem. C, 2015, 119, 25840-25844.

63 K. Ishikawa, T. Yago and M. Wakasa, J. Phys. Chem. C, 2018, 122, 22264-22272.

64 H. Sakai, R. Inaya, H. Nagashima and S. Nakamura, J. Phys. Chem. Lett., 2018, 9, 3354-3360.

65 J. J. Burdett, G. B. Piland and C. J. Bardeen, Chem. Phys. Lett., 2013, 585, 1-10.

66 Z. Yang, Z. Mao, Z. Xie, Z. Y. Zhang, S. Liu, J. Zhao, J. Xu, Z. Chi and M. P. Aldred, Chem. Soc. Rev., 2017, 46, 915-1016.

67 H. Yamagata, J. Norton, E. Hontz, Y. Olivier, D. Beljonne, J. L. Brédas, R. J. Silbey and F. C. Spano, J. Chem. Phys., 2011, 134, 204703.

68 X. Feng, A. V. Luzanov and A. I. Krylov, J. Phys. Chem. Lett., 2013, 4, 3845-3852.

69 K. Aryanpour, A. Shukla and S. Mazumdar, J. Phys. Chem. C, 2015, 119, 6966-6979.

70 A. J. Musser and J. Clark, Annu. Rev. Phys. Chem., 2019, 70, 323-351.

71 D. Beljonne, H. Yamagata, J. L. Brédas, F. C. Spano and Y. Olivier, Phys. Rev. Lett., 2013, 110, 226402.

72 A. A. Bakulin, S. E. Morgan, T. B. Kehoe, M. W. B. Wilson, A. W. Chin, D. Zigmantas, D. Egorova and A. Rao, Nat. Chem., 2016, 8, 16-23.

73 R. E. Merrifield, Pure Appl. Chem., 1971, 27, 481-498.

74 H. Nagashima, S. Kawaoka, S. Akimoto, T. Tachikawa, Y. Matsui, H. Ikeda and Y. Kobori, J. Phys. Chem. Lett., 2018, 9, 5855-5861.

75 T. J. Penfold, E. Gindensperger, C. Daniel and C. M. Marian, Chem. Rev., 2018, 118, 6975-7025.

76 D. M. E. Freeman, A. J. Musser, J. M. Frost, H. L. Stern, A. K. Forster, K. J. Fallon, A. G. Rapidis, F. Cacialli, I. McCulloch, T. M. Clarke, R. H. Friend and H. Bronstein, J. Am. Chem. Soc., 2017, 139, 11073-11080.

77 Y. Y. Cheng, B. Fückel, T. Khoury, R. G. C. R. Clady, M. J. Y. Tayebjee, N. J. Ekins-Daukes, M. J. Crossley and T. W. Schmidt, J. Phys. Chem. Lett., 2010, 1, 1795-1799.

78 B. H. Wallikewitz, D. Kabra, S. Gélinas and R. H. Friend, Phys. Rev. B: Condens. Matter Mater. Phys., 2012, 85, 045209. 